\title{
La qualità della comunicazione emotiva come strumento di integrazione relazionale e affettiva nei luoghi dell'educazione e del prendersi cura
}

\section{Simona Pelliciari}

\author{
(2) OpenEdition \\ Journals \\ Edizione digitale \\ URL: http://journals.openedition.org/esp/946 \\ DOI: $10.4000 /$ esp.946 \\ ISSN: 2532-0319 \\ Editore \\ Centre d'Information sur l'Éducation Bilingue et Plurilingue

\section{Edizione cartacea} \\ Data di pubblicazione: 1 dicembre 2016 \\ Paginazione: 71-73 \\ ISSN: 1127-266X \\ Notizia bibliografica digitale \\ Simona Pelliciari, « La qualità della comunicazione emotiva come strumento di integrazione \\ relazionale e affettiva nei luoghi dell'educazione e del prendersi cura », Éducation et sociétés plurilingues \\ [Online], 41 | 2016, Messo online il 20 octobre 2017, consultato il 15 septembre 2020. URL : http:// \\ journals.openedition.org/esp/946
}




\section{LA QUALITÀ DELLA COMUNICAZIONE EMOTIVA COME STRUMENTO DI INTEGRAZIONE RELAZIONALE E AFFETTIVA NEI LUOGHI DELL'EDUCAZIONE E DEL PRENDERSI CURA}

\section{Simona Pelliciari}

L'expérience professionnelle d'une éducatrice sociale montre que la capacité de l'écoute et l'empathie sont les facteurs positifs les plus importants pour construire une relation "sensibile" et efficace basée sur l'acceptation et la confiance réciproques.

Mots-clés: jeunes adultes handicapés, travail social, relation, empathie, communication affective, patois

A social worker's professional experience demonstrates how empathy and the ability to listen are the most important positive factors in building a "sensitive" and efficient relationship based on reciprocal acceptance and trust.

Key-zords: handicapped young adults, social work, relationship, empathy, sensitive communication, dialect

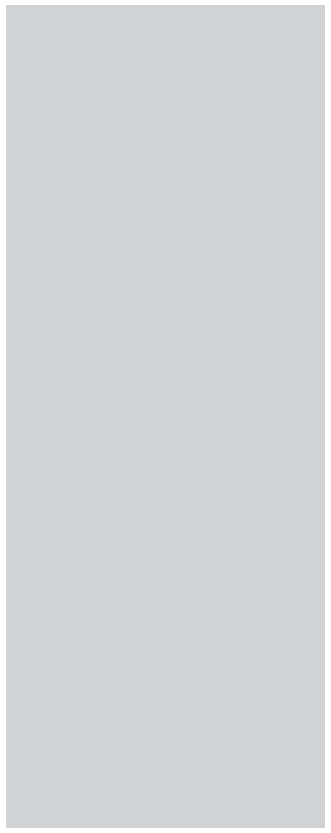

$\mathrm{M}$ i chiamo Simona Pelliciari, lavoro da 18 anni con persone giovani-adulti disabili inseriti nei centri diurni dei servizi sociali del comune di Modena. Mi sono formata come operatrice socio sanitaria-assistenziale e in seguito come educatrice sociale.

La mia esperienza relazionale con persone che non possono avere una comunicazione verbale e gestuale completa o quantomeno efficace, mi ha sottoposto a diversi interrogativi sia di natura etico-morale che di natura tecnico-linguistica. Di fronte ad un bisogno non espresso, di fronte alla chiusura comunicativa come provare a costruire una relazione che sia centrata sulla persona, tenendo conto dei bisogni, paure, emozioni soggettivi di una identità personale?

Da dove cominciare un percorso di comunicazione che consenta ad entrambi di realizzare per quel che possibile un progetto di vita di socialità e di presenza?

Investire su apprendimento e contenuti tecnici appare fuori luogo e superato. Esclusi perché, quando il prendersi cura è un farsi carico della persona in forte compromissione comunicativarelazionale, occorrono risorse comunicative umane spendibili in qualunque luogo e fase della vita. Per questo forse la relazione empatica è uno strumento fondante per la possibilità di interagire 
La qualità della comunicazione emotiva S. Pelliciari insieme. L'empatia è la capacita degli esseri umani di mettersi nei panni dell'altro, facendo una vera e propria esperienza di ciò che l'altro sta provando o soffrendo o gioendo. L'esperienza è la parola chiave, esperienza individuale soggettiva che ognuno costruisce in base alla propria dimensione bio-psico-sociale-spirituale.

La relazione empatica è provare ciò che prova l'altro, le sue paure, le sue emozioni, il suo contesto culturale, le sue tradizioni, i rituali simbolici e comunicativi che orientano spesso atteggiamenti, aperture e resistenze di un contesto sociale... Significa fare esperienza di vedere con gli occhi dell'altro e sentire con le orecchie dell'altro... Provare la chiusura emotiva dell'altro...Spesso mi è accaduto di non riuscire a provocare nella persona in difficoltà nessun tipo di reazione comunicativa, nessuna espressione di sé. Questo blocco mi ha portato a ricercare metodologie, anche creative, ma ho riscontrato che la valorizzazione dello strumento dell'empatia, mi ha sempre aiutato nel costruire una relazione di fiducia. Per esempio provando a ricreare una comunicazione simbolica, come un suono, un intercalare verbale, un modo di dire territoriale, dialettale, accompagnato da una gestualità tipica del contesto esperienziale della persona in difficoltà comunicativa. La speranza che la memoria emozionale affettiva venga attivata e risuoni nell'altra persona, creando una base relazionale di fiducia e di accettazione positiva incondizionata in cui l'altro si senta accolto e riconosciuto.

Per esempio in alcuni momenti difficili di chiusura comunicativa totale, e di fronte alla evidente sofferenza di una ragazza che presentava disturbi dello spettro autistico, ho utilizzato dei modi di dire dialettali che sono tipici del contesto socio-culturale territoriale della tradizione contadina della profonda campagna modenese, territorio di provenienza della ragazza prima di essere inserita nel servizio del centro diurno. Questo ha raccolto in una breve comunicazione tutta l'esperienza emotiva dell'infanzia della persona in difficoltà, che si è poi tradotta in uno sblocco, prima con un pianto poi con una reazione di richiesta nei miei confronti, costituendo il primo approccio relazionale e funzionale tra noi due.

Questa riflessione sul comprendere il campo esperienziale e in questo caso il contesto bio-psico-sociale e spirituale di provenienza è fondamentale per conoscere non solo l'origine di provenienza ma il campo fenomenologico soggettivo che ognuno elabora individualmente. In una dimensione di integrazione dell'alterità e di relazioni funzionali, l'empatia, l'esperienza soggettiva la comunicazione emotiva-affettiva possono auspicarsi come strumenti 
La qualità della comunicazione emotiva

S. PelLiciarI
RIFERIMENTO importanti nelle difficoltà di qualunque natura, sia di origine culturale, sia di comportamento che di comunicazione. Oggi più che mai occorre che nei contesti educativi, sanitari, assistenziali l'importanza di favorire una comunicazione emozionale, affettiva, sia condivisa e sempre più valorizzante e qualificante.

Anche le neuroscienze, con la scoperta dei Neuroni Specchio (Gallese \& Rizziolatti et alii. 2006), ci hanno dimostrato (dopo una prima intuizione osservando una scimmia in laboratorio alla università di Parma) che gli esseri umani sono in possesso delle competenze innate di empatia, hanno le abilità di mettersi negli occhi dell'altro e di vedere e sentire come lui pur continuando ad essere se stessi...

I neuroni specchio sono una classe di neuroni che si attivano quando un individuo compie un'azione e quando l'individuo osserva la stessa azione compiuta da un altro soggetto. È stato provato che osservando un soggetto il quale veniva sottoposto a una risonanza magnetica funzionale gli si illuminavano le stesse aree celebrali rispetto al soggetto che stava compiendo l'esperienza.

Nelle professioni educative e del prendersi cura occorre allenare, valorizzare, espandere questa prodigiosa, innata risorsa dell'essere umano.

RIZZOLATTI G., FOGASSI L., GALLESE V. 2006. Mirrors of the Mind. Scientific American 295: 54-61. 